\section{Auto-rotação cefálica ativa em pacientes com tontura/ vertigem}

\section{Active cephalic auto- rotation in patients with dizziness/ vertigo}

\author{
Aída R.M. de Assunção ${ }^{1}$, Sergio Albertino ${ }^{2}$, \\ Marco A. M. T. Lima
}

Resumo / Summary

Introdução: A auto-rotação cefálica ativa é um teste rápido, de simples realização, não invasivo, que não causa desconforto ao paciente, pode ser realizado com facilidade em crianças e avalia o reflexo vestíbulo-ocular nas freqüências fisiológicas de movimentação da cabeça, utilizadas na vida cotidiana (de 1 a $4 \mathrm{~Hz}$ ). Forma de estudo: clínico retrospectivo não randomizado. Objetivo: Avaliar pacientes com queixas de tontura/vertigem através da auto-rotação cefálica ativa comparando os sintomas com as alterações encontradas. Material e método: Um grupo de 1281 pacientes com queixa de tontura/vertigem foi submetido ao teste de auto-rotação cefálica ativa horizontal com alvo fixo, como uma etapa da avaliação otoneurológica. Resultados: As idades variaram de três a 93 anos com média de 49,6 anos, sendo 946 (73,8\%) do sexo feminino e $335(26,2 \%)$ do sexo masculino. A queixa de vertigem foi relatada por $896(69,9 \%)$ dos pacientes e a tontura por 385 (30,1\%). A faixa de freqüência de resposta à prova de auto-rotação cefálica variou de 1,5 a $7,5 \mathrm{~Hz}$ com média de $3,5 \mathrm{~Hz}$. A prova de auto-rotação cefálica ativa foi normal em 937 (73,1\%) e alterada em 344 (26,9\%) dos pacientes. As alterações mais freqüentes foram as relacionadas ao ganho (aumento, redução) isoladas ou associadas a alterações de fase e simetria, em 241 (19\%) pacientes. O aumento do ganho isolado foi verificado em 92 pacientes (7,2\%). Conclusão: Não houve relação entre as alterações na prova de auto-rotação cefálica ativa horizontal e as queixas de tontura e vertigem.
Palavras-chave: auto-rotação cefálica, tontura, vertigem. Key words: cephalic auto-rotation test, dizziness, vertigo. ntroduction: Active cephalic auto-rotation is a rapid, easy, painless and comfort test, that can be done in children and evaluate the vestibulo-ocular reflex in the physiologic frequencies of head, used in the every day life (from one to four hertz). Study design: clinical retrospective not randomized. Aim: Evaluate patients with complaints of dizziness/vertigo through the active cephalic auto-rotation comparing symptoms with alterations found. Material and method: A group of 1281 patients with dizziness underwent the horizontal active cephalic auto-rotation test with fixed target, as a part of otoneurological evaluation to verify the alterations that showed up. Results: Ages rate from three to 93 years with average of 49,6 years, being 946 (73,8\%) females and 335 (26,2\%) males. The vertigo complaint was reported by $896(69,9 \%)$ patients and dizziness by 385 (30,1\%). The frequency answering rate to cephalic auto-rotation test ranged from 1,5 to $7,5 \mathrm{~Hz}$ with average of $3,5 \mathrm{~Hz}$. The active cephalic auto-rotation test was normal in $937(73,1 \%)$ and abnormal in 344 (26,9\%) patients. The most frequent alterations were those having to do with the gain (increase - reduction) isolated or associated to alterations of phase and symmetry in 241 (19\%) patients. The increase of gain isolated was verified in $92(7,2 \%)$ patients. Conclusion: There was no relation between alterations in the active cephalic auto-rotation test and the complaints of dizziness and vertigo.

\footnotetext{
${ }^{1}$ Prof. Assistente da Universidade do Estado do Rio de Janeiro (UERJ)

${ }^{2}$ Prof. Adjunto da Universidade Federal Fluminense (UFF)

${ }^{3}$ Prof. Adjunto da Universidade Federal do Rio de Janeiro (UFRJ)

Universidade Federal do Rio de Janeiro(UFRJ)

Endereço para correspondência: Aída Regina Monteiro de Assunção - Rua Mem de Sá 186, Icaraí, Niterói, RJ - 24220-261

Fone/fax (0xx21) 7104278 - e-mail: aidaregina@openlink.com.br

Dissertação apresentada à Universidade Federal do Rio de Janeiro para obtenção do título de Mestre em Otorrinolaringologia em dezembro de 2000. Trabalho selecionado para premiação, área de otologia, apresentado em 26/08/01 no II Congresso Triológico de Otorrinolaringologia em Goiânia - GO Artigo recebido em 18 de setembro de 2001. Artigo aceito em 15 de outubro de 2001.
} 


\section{INTRODUÇÃO}

A auto-rotação cefálica ativa, também denominada auto-rotação vestibular ou balanço cefálico ativo, é um teste rápido, de simples realização, não invasivo, que não causa desconforto ao paciente, pode ser realizado com facilidade em crianças e avalia o reflexo vestíbulo-ocular (RVO) nas freqüências fisiológicas de movimentação da cabeça, utilizadas na vida cotidiana (de $1 \mathrm{a} 4 \mathrm{~Hz})^{9,5,13}$.

As provas tradicionais de avaliação do RVO, como os testes calóricos e as cadeiras rotatórias, utilizadas em conjunto com a eletronistagmografia (ENG), vectoeletronistagmografia (VENG) ou eletronistagmografia computadorizada, empregam freqüências baixas de estimulação (entre $0,003$ e $2 \mathrm{~Hz})^{17,9}$ muito diferentes das freqüências fisiológicas, e utilizam estímulos obtidos em condições não-naturais (irrigação do conduto auditivo externo com água ou ar, rotação de todo corpo) gerando desta forma um grande percentual de exames normais em pacientes com disfunção vestibular ${ }^{2,16}$.

A alta prevalência das queixas de distúrbios do equilíbrio corporal associada a resultados normais nos testes vestibulares empregados na rotina de avaliação otoneurológica justifica o estudo através de exames complementares mais precisos, com o objetivo de identificar o grau de comprometimento e a participação do labirinto na gênese da tontura.

O RVO tem por objetivo estabilizar a imagem na retina e manter a visão clara e o equilíbrio corporal durante a locomoção e a movimentação habitual da cabeça. Para isso os movimentos oculares devem ser iguais e opostos aos da cabeça. Distúrbios na resposta do RVO geram alterações do equilíbrio corporal que são a segunda queixa mais freqüente na clínica do otorrinolaringologista, clínico geral e neurologista em pacientes até 65 anos e a mais freqüente após os 65 anos. Cerca de $65 \%$ dos idosos até 70 anos e $75 \%$ acima dos 70 anos apresentam sintomas de distúrbios do equilíbrio ${ }^{21}$.

O equilíbrio corporal é mantido em função da interação de diversos sistemas (vestibular, visual, propriceptivo), sob a coordenação do cerebelo. As informações provenientes desses sistemas devem ser coerentes. Quando existem informações conflitantes o resultado é um distúrbio do equilíbrio corporal ${ }^{6}$.

As queixas mais freqüentes relacionadas ao equilíbrio corporal são tontura e vertigem. A tontura é a sensação de alteração do equilíbrio corporal de caráter não-rotatório. A vertigem é a tontura de caráter rotatório, quando o paciente tem a sensação que seu corpo ou os objetos ao seu redor estão girando ${ }^{10}$.

A proposta deste trabalho é avaliar os pacientes com tontura e vertigem através do teste de auto-rotação cefálica ativa horizontal com alvo fixo, comparando estes sintomas com as prováveis alterações encontradas.

\section{PACIENTES E MÉTODO}

Foram avaliados os prontuários de todos os pacientes submetidos ao teste de auto-rotação cefálica ativa horizontal com alvo fixo, no período de janeiro de 1998 a dezembro de 1999 na Clínica Otorrinos Reunidos Ltda., no total de 1902 pacientes. Foi pesquisada a idade, sexo, queixa principal, resultado da avaliação audiométrica, faixa de freqüência da movimentação cefálica, alterações presentes à prova de auto-rotação cefálica ativa horizontal, sintomas durante a realização do exame. Foram incluídos nesta pesquisa os pacientes com queixa de tontura (queixas não-rotatórias) ou vertigem (queixas rotatórias), totalizando 1281 exames. Excluímos 621 pacientes que apresentavam outras queixas como zumbido ou hipoacusia.

O trabalho foi retrospectivo, garantindo-se a confidencialidade dos dados e o anonimato dos pacientes. Foi aprovado pelo Comitê de Ética em Pesquisa (CEP) do Hospital Clementino Fraga Filho da Universidade Federal do Rio de Janeiro em 10/06/99, com protocolo de número 53/99-CEP.

A aparelhagem utilizada foi o VORTEQ (Vestibular Ocular Reflex Test Equipment) fabricado pela MICRO MEDICAL TECHNOLOGIES, parte integrante do Sistema de Nistagmografia Computadorizada META 4 CHANNEL ULTRA COMPUTERIZED ENG Versão 4.5, instalado em um computador COMPAQ DESKPRO PENTIUM II, acoplado a uma impressora HP (HEWLETT PACKARD) LASER JET 6L.

O exame de auto-rotação cefálica ativa horizontal com alvo fixo foi realizado com o paciente confortavelmente sentado, em ambiente semi-escuro. A pele foi previamente limpa com gaze embebida em álcool para remoção de impurezas e colocação dos eletrodos (dois ativos, um no canto externo periorbitário direito e o outro no esquerdo e um eletrodo neutro, colocado na linha média frontal). O sensor de velocidade angular, na posição vertical, ficou firmemente ajustado através de uma coroa, à cabeça do paciente, que foi orientado a permanecer de olhos abertos observando um ponto luminoso estacionário distante $100 \mathrm{~cm}$ a sua frente e a movimentar a cabeça para a direita e para a esquerda, com uma amplitude de rotação angular de aproximadamente $10^{\circ}$ acompanhando um sinal acústico de freqüência variável produzido por um metrônomo eletrônico acoplado ao equipamento.

A primeira estimulação foi realizada na freqüência de $1 \mathrm{~Hz}$, depois iniciou-se com $1 \mathrm{~Hz}$ até $3 \mathrm{~Hz}$ e a última estimulação de $1 \mathrm{~Hz}$ até $5 \mathrm{~Hz}$, cada seqüência com duração de 15 segundos, podendo a movimentação cefálica do paciente atingir até $7,5 \mathrm{~Hz}$. O computador selecionou os ciclos de rotação da cabeça durante o teste que foram agrupados de acordo com a freqüência. Todos os ciclos próximos a $1 \mathrm{~Hz}(\mathrm{de} 0,5 \mathrm{a} 1,5 \mathrm{~Hz}$ ) foram colocados em um grupo os próximos a $2 \mathrm{~Hz}$ em outro e assim sucessiva- 
mente ${ }^{8,2,4,13}$. O paciente foi treinado antes de se iniciar o registro do teste. As faixas de freqüência do exame foram determinadas pela movimentação da cabeça do paciente e o programa computadorizado permite a análise de freqüências na faixa de 0,5 a $7,5 \mathrm{~Hz}$.

O padrão de normalidade utilizado foi o que vem determinado na aparelhagem, e os resultados foram facilmente observados através de gráficos na tela do monitor, onde a área entre as duas linhas contínuas no gráfico representou os limites normais. A linha superior representou a média mais dois desvios padrões e a inferior a média menos dois desvios padrões ${ }^{8,2,13}$.

Os parâmetros de avaliação do RVO na prova de auto-rotação cefálica ativa foram: ganho, definido como a relação entre a velocidade dos olhos e da cabeça (relação entre a intensidade do estímulo e da resposta); fase, analisada com relação ao atraso ou avanço da resposta ocular em relação a movimentação da cabeça (relação angular entre a curva do estímulo e da resposta) e simetria, comparação entre os ganhos dos movimentos cefálicos de direções opostas ${ }^{13,14}$.

O exame é indolor e sem riscos. O paciente pode sentir tontura, náuseas e cefaléia, por esta razão foi orientado a realizar o teste em jejum de quatro horas, suspender medicação (ansiolíticos, tranqüilizantes, hipnóticos, analgésicos e depressores labirínticos) nas setenta e duas horas precedentes ao exame e alimentação (café, chás, achocolatados, bebidas alcoólicas, refrigerantes), e fumo, vinte e quatro horas antes para que estes não interferissem nas respostas do labirinto. Caso utilizasse medicações tipo anticonvulsivantes foi orientado a informar-se com o neurologista sobre a possibilidade de suspensão. Os exames foram realizados fora do período da crise.

A fim de medir o grau de correlação entre as variáveis da amostra utilizou-se a técnica não paramétrica Coeficiente de Contingência (C) e aplicou-se a prova de significância $X^{2}$ (Qui-quadrado), adotando-se sobre este coeficiente o nível de significância de $0,05(\mathrm{p}<0,005)$, sendo 95\% de certeza para afirmativas e/ou negativas que este estudo viesse a denotar.

As faixas etárias foram distribuídas em intervalos, permitindo que fossem apresentadas de forma contínua, sem superposição, de tal modo que cada valor do conjunto de observação possa ser alocado em um, e apenas um dos intervalos, segundo a fórmula de Sturges que possibilita uma distribuição mais uniforme do número de indivíduos em cada intervalo.

\section{RESULTADOS}

\section{Características da amostra}

Foram analisados os prontuários de 1281 pacientes com queixa de tontura ou vertigem que realizaram a prova de auto-rotação cefálica ativa horizontal com alvo fixo, no período de janeiro de 1998 a dezembro de 1999.

A idade dos pacientes variou entre três e 93 anos, com média de 49,6 anos, desvio padrão (D.P.) de 16,8 distribuídos de acordo com a Tabela 1.

\section{Características dos sintomas relatados pelos pacientes na anamnese}

A queixa de vertigem foi mais freqüente, $69,9 \%$ (896), enquanto a tontura foi descrita por $30,1 \%$ (385) (Tabela 3).

\section{Resultados na prova de auto-rotação cefálica ativa horizontal com alvo fixo}

A faixa de freqüência de resposta da prova de autorotação cefálica variou de 1,5 a 7,5Hz com média de $3,5 \mathrm{~Hz}$, desvio padrão de 1,2 (Tabela 4).

\section{Correlação entre alterações na prova de auto-rotação cefálica ativa horizontal com alvo fixo e queixa tontura/vertigem}

Ao cruzarmos os dados de alteração na prova de auto-rotação cefálica ativa com queixa de vertigem e tontura (Tabela 9), verificamos que o percentual de pacientes com queixa de vertigem e prova de auto-rotação normal (659 pacientes - 73,55\%) foi semelhante ao de pacientes com queixa de tontura e auto-rotação normal (278 pacientes - 72,20\%). A mesma proporção ocorreu quando comparamos a queixa com alteração na prova de autorotação, $26,45 \%$ de pacientes com vertigem e $27,80 \%$ de pacientes com tontura apresentaram alteração.

A prova de significância não permitiu rejeitar a hipótese de nulidade, indicando dessa forma que quaisquer diferenças podem ser consideradas como variações casuais esperadas em uma amostra aleatória da população, o que não representa a existência de um relacionamento entre as variáveis na população da qual se extraiu a amostra. Percebe-se que o valor de Coeficiente de contingência é muito baixo, indicando uma correlação próxima de zero $(\mathrm{C}=0,01)$.

\section{Correlação entre alteraçôes na prova de auto-rotação cefálica ativa horizontal com alvo fixo e sexo}

A proporção entre exames normais e alterados foi semelhante nos pacientes do sexo feminino e masculino (Tabela 10).

A prova de significância não permitiu rejeitar a hipótese de nulidade, os valores observados foram muito próximos dos valores esperados, indicando dessa forma que as diferenças podem ser consideradas como variações casuais esperadas em uma amostra aleatória da população, o que não representa a existência de um relacionamento entre as variáveis na população da qual se extraiu a amostra $(\mathrm{C}=0,04)$. 
Tabela 1. Distribuição de faixa etária da amostra

\begin{tabular}{lcc}
\hline Faixa etária & Freqüência Absoluta & Freqüência Relativa \\
\hline 2 a 10 anos & 21 & $1,6 \%$ \\
11 a 19 anos & 25 & $2,0 \%$ \\
20 a 28 anos & 93 & $7,3 \%$ \\
29 a 37 anos & 179 & $14,0 \%$ \\
38 a 46 anos & 244 & $19,0 \%$ \\
47 a 55 anos & 227 & $17,7 \%$ \\
56 a 64 anos & 203 & $15,8 \%$ \\
65 a 73 anos & 194 & $15,1 \%$ \\
74 a 82 anos & 81 & $6,3 \%$ \\
83 a 91 anos & 13 & $1,0 \%$ \\
Acima de 92 anos & 1 & $0,1 \%$ \\
\hline Total & 1281 & $100,0 \%$ \\
\hline
\end{tabular}

Tabela 2. Proporção entre os sexos

\begin{tabular}{lcc}
\hline Sexo & Freqüência Absoluta & Freqüência Relativa \\
\hline Feminino & 946 & $73,8 \%$ \\
Masculino & 335 & $26,2 \%$ \\
\hline Total & 1281 & $100,0 \%$ \\
\hline
\end{tabular}

Tabela 3. Proporção entre as queixas de vertigem e tontura

\begin{tabular}{lcc}
\hline Queixa & Freqüência Absoluta & Freqüência Relativa \\
\hline Vertigem & 896 & $69,9 \%$ \\
Tontura & 385 & $30,1 \%$ \\
\hline Total & 1281 & $100,0 \%$ \\
\hline
\end{tabular}

Tabela 4. Faixa de freqüência de resposta no prova de autorotação cefálica ativa

\begin{tabular}{lcccccc}
\hline Variável & $\mathrm{N}$ & Média & D.P & Mínimo & Máximo & Moda \\
\hline Faixa & 1281 & 3,5 & 1,2 & 1,5 & 7,5 & 2,5 \\
\hline
\end{tabular}

Tabela 5. Proporção de exames normais e alterados

\begin{tabular}{lcc}
\hline & Freqüência Absoluta & Freqüência Relativa \\
\hline Normal & 937 & $73,1 \%$ \\
Alterado & 344 & $26,9 \%$ \\
\hline Total & 1281 & $100,0 \%$ \\
\hline
\end{tabular}

\section{DISCUSSÃO}

\section{Relação entre os sexos}

Em 1281 pacientes avaliados, 946 eram do sexo feminino (73,8\%) e 335 masculino (26,2\%) (Tabela 2), demonstrando que as queixas de tontura e vertigem são
Tabela 6. Distribuição entre exames normais e alterados

\begin{tabular}{lcc}
\hline & \multicolumn{2}{c}{ Freqüência } \\
\cline { 2 - 3 } & Absoluta & Relativa \\
\hline Normal & 937 & $73,1 \%$ \\
Aumento ganho & 92 & $7,2 \%$ \\
Avanço fase & 31 & $2,4 \%$ \\
Aumento ganho+assimetria esquerda & 29 & $2,3 \%$ \\
Assimetria esquerda & 20 & $1,6 \%$ \\
Aumento ganho+avanço fase & 16 & $1,2 \%$ \\
Atraso fase & 15 & $1,2 \%$ \\
Aumento ganho+atraso fase+assimetria esq & 14 & $1,1 \%$ \\
Redução ganho & 14 & $1,1 \%$ \\
Redução ganho+assimetria esquerda & 11 & $0,9 \%$ \\
Avanço fase+assimetria esquerda & 9 & $0,7 \%$ \\
Redução ganho+avanço fase+assimetria direita & 9 & $0,7 \%$ \\
Assimetria direita & 7 & $0,5 \%$ \\
Aumento ganho+avanço fase+assimetria esq & 7 & $0,5 \%$ \\
Atraso fase+assimetria direita & 6 & $0,5 \%$ \\
Atraso fase+assimetria esquerda & 6 & $0,5 \%$ \\
Avanço fase+assimetria direita & 6 & $0,5 \%$ \\
Aumento ganho+assimetria direita & 5 & $0,4 \%$ \\
Aumento ganho+atraso fase+assimetria direita & 5 & $0,4 \%$ \\
Redução ganho+atraso fase+assimetria direita & 5 & $0,4 \%$ \\
Aumento ganho+assimetria direita/esquerda & 4 & $0,3 \%$ \\
Aumento ganho+atraso fase & 4 & $0,3 \%$ \\
Redução ganho+assimetria direita & 4 & $0,3 \%$ \\
Redução ganho+atraso fase+assimetria esq & 4 & $0,3 \%$ \\
Aumento e redução ganho+atraso fase & 3 & $0,2 \%$ \\
Redução ganho+avanço fase & 3 & $0,2 \%$ \\
Aumento ganho+atraso fase+assimetria esq/dir & 2 & $0,2 \%$ \\
Aumento/redução ganho & 2 & $0,2 \%$ \\
Redução ganho+atraso fase & 2 & $0,2 \%$ \\
Assimetria direita/esquerda & 2 & $0,2 \%$ \\
Aumento ganho+avanço fase+assimetria dir & 1 & $0,1 \%$ \\
Aumento redução ganho+atraso fase & 1 & $0,1 \%$ \\
Aumento/red. Ganho+atraso fase+ass dir/esq & 1 & $0,1 \%$ \\
Aumento/redução ganho+avanço fase+ass esq & 1 & $0,1 \%$ \\
Avanço fase+assimetria esquerda direita & 1 & $0,1 \%$ \\
Redução ganho+avanço fase+assimetria esq & 1 & $0,1 \%$ \\
Redução ganho+avanço atraso fase+ass. dir & 1 & $0,1 \%$ \\
\hline Total & 1281 & $100,0 \%$ \\
\hline
\end{tabular}

Tabela 7. Proporção de pacientes com e sem sintomas durante o exame

\begin{tabular}{lcc}
\hline Pacientes & Freqüência Absoluta & Freqüência Relativa \\
\hline Com sintomas & 684 & $53,4 \%$ \\
Sem sintomas & 597 & $46,6 \%$ \\
\hline Total & 1281 & $100,0 \%$ \\
\hline
\end{tabular}

mais comuns no sexo feminino. Esses dados estão de acordo com os obtidos por outros trabalhos pesquisados. Caovilla e col. ${ }^{7}$ e Ganança e col. ${ }^{12}$ referem que as tonturas mostram maior predileção pelo sexo feminino na proporção de 2:1.

Maudonnet e col..$^{22}$, avaliando 4825 pacientes, 
Tabela 8. Proporção dos sintomas durante o exame

\begin{tabular}{lcc}
\hline $\begin{array}{l}\text { Sintomas durante } \\
\text { o exame }\end{array}$ & $\begin{array}{c}\text { Freqüência } \\
\text { Absoluta }\end{array}$ & $\begin{array}{c}\text { Freqüência } \\
\text { Relativa }\end{array}$ \\
\hline Tontura & 658 & $51,4 \%$ \\
Náusea+tontura & 14 & $1,1 \%$ \\
Cefaléia & 4 & $0,3 \%$ \\
Cefaléia+tontura & 3 & $0,2 \%$ \\
Náusea & 2 & $0,2 \%$ \\
Náusea+cefaléia+tontura & 1 & $0,1 \%$ \\
Dor coluna & 1 & $0,1 \%$ \\
Sudorese+tontura & 1 & $0,1 \%$ \\
Sem queixas & 597 & $46,6 \%$ \\
\hline Total & 1281 & $100,0 \%$ \\
\hline
\end{tabular}

Tabela 9. Alterações na prova de auto-rotação cefálica ativa e queixa de tontura/vertigem

\begin{tabular}{lccc}
\hline \multirow{2}{*}{ Queixa } & \multicolumn{3}{c}{ Auto-rotação cefálica ativa horizontal } \\
\cline { 2 - 4 } & Normal & Alterada & Total \\
\hline Vertigem & 659 & 237 & 896 \\
Tontura & 278 & 107 & 385 \\
\hline Total & 937 & 344 & 1281 \\
\hline
\end{tabular}

Tabela 10. Alterações na prova de auto-rotação cefálica e sexo

\begin{tabular}{lccc}
\hline \multirow{2}{*}{ Sexo } & \multicolumn{3}{c}{ Auto-rotação cefálica ativa horizontal } \\
\cline { 2 - 4 } & normal & Alterada & Total \\
\hline Feminino & 703 & 243 & 946 \\
Masculino & 234 & 101 & 335 \\
\hline Total & 937 & 344 & 1281 \\
\hline
\end{tabular}

encontraram $3124(64,75 \%)$ do sexo feminino e 1702 $(35,25)$ do sexo masculino.

Caovilla ${ }^{3}$, em um estudo com 1000 pacientes encontrou 625 casos do sexo feminino e 375 do sexo masculino.

Ganança ${ }^{15}$, em um levantamento com 7850 casos consecutivos de vertigem e outros tipos de tontura, avaliados no período de janeiro de 1985 a junho de 2000 , encontrou $4812(61,3 \%)$ do sexo feminino e 3038 (38,7\%) do sexo masculino.

\section{Relação entre as faixas etárias}

Em nossa casuística a faixa etária variou entre três e 93 anos sendo a maior incidência entre 38 e 46 anos (19\%) (Tabela 1), dados semelhantes aos obtidos por outros autores.

Caovilla e col. ${ }^{7}$, em pacientes entre cinco meses e mais de 81 anos, relatam que as tonturas mostraram-se pouco freqüentes dos cinco aos 20 anos $(6,2 \%)$, acometendo preferencialmente a faixa entre 21 e 80 anos (32,3\% entre 21 a 40 anos; 34,7\% entre 41 a 60 anos; $24,6 \%$ entre 61 a 80 anos). A prevalência acima de 81 anos (2,2\%) foi minimizada devido ao pequeno número de pacientes nessa faixa etária.

Maudonnet e col. ${ }^{22}$, avaliando 4825 pacientes com doenças cócleo-vestibulares encontraram o maior percentual de afecções na faixa etária de 40 a 50 anos, 1252 pacientes $(25,95 \%)$, na faixa de 30 a 40 anos o percentual foi de $22,16 \%$, decrescendo para $18,07 \%$ na faixa de 50 a 60 anos.

Caovilla ${ }^{3}$ encontrou a faixa etária variando entre 17 e 84 anos em um estudo com 1000 pacientes.

Ganança $^{15}$ em seu levantamento com 7850 casos encontrou pacientes na faixa etária entre zero e 95 anos. A freqüência maior foi nas faixas entre 31 e 75 anos $(72,25 \%)$ com um percentual maior na faixa de 70 a 75 anos $(9,48 \%)$.

\section{TONTURA E VERTIGEM}

A queixa de vertigem foi informada por $896(69,9 \%)$ dos pacientes e a tontura por 385 (30,1\%) (Tabela 3). Casuística semelhante à encontrada por Caovilla e col.7 em um estudo onde a vertigem foi a mais freqüente das tonturas $(63,7 \%)$. Menos freqüentes em seu estudo foram as tonturas não-rotatórias (22,1\%) e a associação dos dois tipos de tontura em um mesmo paciente (14,2\%).

Bento e col. ${ }^{1}$ encontraram em um estudo com 230 pacientes instabilidade em 58 pacientes e tontura rotatória (vertigem) em 60.

\section{FAIXA DE FREQÜÊECIA}

A faixa de freqüência de resposta da prova de autorotação cefálica variou de 1,5 a 7,5Hz com média de $3,5 \mathrm{~Hz}$, desvio padrão de 1,2 (Tabela 4).

Fineberg e col. ${ }^{9}$ avaliaram 15 pacientes com idades variando entre 19 e 71 anos, e comparando os resultados na luz do dia e em uma sala escura não encontraram diferenças significativas para freqüências entre 2 e $6 \mathrm{~Hz}$.

O'Leary e col. ${ }^{24}$ testaram indivíduos idosos sem patologia vestibular ou coclear e indivíduos jovens saudáveis, utilizando o VAT, com o propósito de comparar os resultados e estabelecer os valores normais em pacientes acima de 65 anos. Comparados com os indivíduos jovens as pessoas idosas obtiveram movimentos cefálicos em geral com velocidades menores que $4 \mathrm{~Hz}$ enquanto os jovens responderam em torno de $6 \mathrm{~Hz}$.

Meulenbroeks e col. ${ }^{23}$, em contraste com outros estudos, encontrou um grande número de indivíduos que não foram capazes de realizar os movimentos cefálicos acima de $4 \mathrm{~Hz}$

\section{AUTO-ROTACÃO CEFÁLICA ATIVA}

$\mathrm{Na}$ prova de auto-rotação cefálica ativa horizontal com alvo fixo, realizada em 1281 pacientes encontramos 344 exames (26,9\%) com alteração (Tabela 5).

As alterações mais freqüente foram as relacionadas 
ao ganho. O aumento isolado do ganho ocorreu em 7,2\% e outras alterações associadas ao ganho 11,8\%, totalizando 241 pacientes (19\%) (Tabela 6).

Tomlinson e col..$^{28}$ avaliaram seis adultos jovens, saudáveis, em diferentes condições de observação de um alvo, não verificando significativa alteração do ganho, nas altas freqüências de rotação da cabeça, no escuro.

Kitsigianis e col. ${ }^{20}$ avaliaram nove pacientes antes e depois do uso de cisplatina e concluíram que o VAT no plano horizontal é eficaz no monitoramento da vestíbulotoxicidade. A redução da performance do RVO observada nestes pacientes é provavelmente decorrente da quimioterapia.

O'Leary e Davis ${ }^{25}$ avaliaram 10 pacientes com quadro agudo de doença de Menière e encontraram achados significativos de aumento do ganho na pesquisa do RVO vertical com VAT. A pesquisa do RVO horizontal estava normal. Verificaram que o exame foi de fácil realização mesmo em pacientes no quadro agudo da doença.

O'Leary e col. ${ }^{24}$ avaliaram dois grupos de indivíduos (jovens e idosos) utilizando o VAT, com o propósito de comparar os resultados e estabelecer os valores normais em pacientes acima de 65 anos. O resultado dos ganhos do RVO horizontal e vertical foi similar nos dois grupos. Para o propósito de análises estatísticas não houve diferenças significativas.

O'Leary e col. ${ }^{26}$, em um estudo com nove pacientes com Schwannoma vestibular, determinaram que as assimetrias acima de 3\% no VAT podem ser um achado útil no diagnóstico do lado e tamanho desse tipo de lesão unilateral.

Murphy ${ }^{21}$ comparou os resultados da eletronistagmografia e do VAT em 102 pacientes com disfunção vestibular com o objetivo de determinar qual o teste seria mais eficaz na avaliação inicial. A ENG parece ser melhor para a avaliação inicial destes pacientes após colhida história, realizado exame físico e audiograma. As limitações do VAT incluem incapacidade para localizar o lado da lesão e para diferenciar lesões periféricas de lesões centrais, mas pode trazer benefícios na testagem de pacientes com vertigem indefinida, trauma e naqueles em que a vertigem não pode ser diagnosticada através da ENG.

Saadat e col. ${ }^{27}$ compararam os resultados da autorotação cefálica com os testes calóricos com duas temperaturas em 39 pacientes com alterações vestibulares periféricas e $10 \mathrm{com}$ Schwannoma vestibular. O resultado deste estudo sugere que a auto-rotação cefálica ativa é mais sensível que a prova calórica. Nos casos de Schwannoma vestibular, todos apresentaram alteração na auto-rotação cefálica ativa, mesmo aqueles em que a origem era no nervo vestibular inferior e apresentavam o teste calórico normal. O teste calórico convencional avalia a via do RVO apenas no canal horizontal e a baixas freqüências, enquanto a auto-rotação cefálica ativa, quando utilizada para pesquisa do RVO horizontal e vertical, avalia todos os três canais, nas freqüências fisiológicas de movimentação da cabeça.

Meulenbroeks e col. ${ }^{23}$ realizaram o VAT em 33 pacientes com o objetivo de investigar a reprodutibilidade e aplicabilidade clínica do método e quantificar o ganho e a fase do RVO em pacientes normais. Concluíram que o teste é preciso e reprodutível na testagem do RVO para altas freqüências.

Caovilla ${ }^{2}$ avaliou 438 pacientes com quadros vertiginosos e hipótese diagnóstica de doença vestibular crônica, através de vecto-eletronistagmografia e a prova de auto-rotação cefálica ativa no plano horizontal com alvo fixo. Neste grupo, 258 casos (58,9\%) apresentaram alterações na VENG e em 180 casos nenhuma anormalidade foi detectada. A prova de auto-rotação cefálica ativa no plano horizontal com alvo fixo, realizada no grupo de pacientes sem alteração à vectoeletronistagmografia, mostrou resultados normais em 95 casos $(52,8 \%)$ e achados anormais em 85 casos $(47,2 \%)$.

Hirvonen e col. ${ }^{19}$ testaram 125 pacientes com autorotação cefálica horizontal com alvo fixo, utilizando o HART (Head auto-rotation test) mostrando que o teste é rápido, confortável e pode ser utilizado para pesquisa do RVO.

Hirvonen e col. ${ }^{18}$, testando pessoas idosas, encontraram alterações em $86 \%$ dos casos. Mais da metade dos pacientes não foi capaz de realizar movimentos rápidos da cabeça, para alcançar altas freqüências. Estes achados podem explicar a alta prevalência de tonturas e quedas no idoso.

Segundo Caovilla e col.7, as alterações da autorotação cefálica ativa como único achado constituíram 16\% da casuística. Quando associada à vertigem e/ou nistagmo de posição observou-se $48,2 \%$ de alteração à rotação cefálica ativa como únicos achados à vestibulometria. Nas vestibulometrias, 33,6\% não apresentaram alterações. Este número reduz-se para $17 \%$ quando realizamos os testes vestibulares associados à auto-rotação cefálica ativa. A autorotação cefálica ativa complementa a vectoeletronistagmografia e a nistagmografia computadorizada e pode detectar alterações em pacientes vertiginosos sem anormalidades nestes exames.

Para Ganança e col. ${ }^{14}$ o ganho é uma medida direta da sensitividade do RVO. Ganhos baixos indicam movimentos oculares subcompensados, enquanto ganhos altos representam movimentos oculares supercompensados. A fase reflete a latência entre picos de velocidade dos olhos em relação aos da cabeça. Ganho e fase anormais provocam sensação de movimento do campo visual, osciloscopia e vertigem. As alterações do ganho (aumento e redução) foram mais encontradas em relação ao RVO horizontal. Concordância da direção da assimetria com o lado da lesão vestibular foi verificada em $62,9 \%$ dos 
pacientes e 20,8\% apresentaram anormalidade apenas a prova de auto-rotação cefálica. A prova de auto-rotação proporciona importante informação adicional sobre o estado funcional do RVO, apesar de não diferenciar os estágios das doenças vestibulares agudas e crônicas, não diferenciar disfunções periféricas de centrais, não distinguir o lado da lesão e não apresentar anormalidades específicas para nenhuma doença vestibular.

A auto-rotação cefálica é um instrumento útil para avaliar os resultados nos programas de monitorização do tratamento dos pacientes vertiginosos. ${ }^{3}$

Nossa casuística, onde as alterações relacionadas ao ganho foram as mais freqüentes, está de acordo com a de outros autores.

\section{SINTOMAS DURANTE O TESTE}

Em nossa amostra, 53,5\% dos pacientes apresentaram sintomas durante a realização da prova de auto-rotação cefálica ativa, sendo o mais freqüente a tontura isolada, com 658 pacientes $(51,4 \%)$ (Tabela 7 ). A queixa de náusea foi relatada por dois pacientes $(0,2 \%)$ e a náusea associada à tontura por 14 (1,1\%) (Tabela 8 ). Em nenhum caso o sintoma foi mais intenso que o apresentado durante a crise, não houve necessidade de interromper o exame e a melhora foi rápida.

O'Leary e Davis ${ }^{25}$ referem que nenhum paciente relatou náuseas ou desconforto, após o término do teste, que durasse mais que alguns minutos, embora esses sintomas sejam comuns e permaneçam após longo tempo quando são realizados os testes calóricos.

Para Ganança e col. ${ }^{14}$ a presença de tontura e o enjôo durante a prova é característica dos pacientes com distúrbios vestibulares.

\section{CONCLUSÕES}

Não houve correlação significativa entre os achados na prova de auto-rotação cefálica ativa horizontal com alvo fixo nos pacientes com queixa de tontura e vertigem.

Independente da idade do paciente não encontramos dificuldade para realização da prova de auto-rotação cefálica ativa horizontal.

As alterações mais freqüentes encontradas foram aquelas relacionadas ao ganho, isoladas ou associadas.

\section{REFERÊNCIAS BIBLIOGRÁFICAS}

1. BENTO, R.F. et al. - Sintomas Vestibulares e Alterações no exame eletronistagmográfico: Estudo de 230 casos. Rev. Bras. Otorrinolaringol., 64(4):397-403, 1998.

2. CAOVIllA, H.H. - Da Rotação Cefálica Ativa em Pacientes Vertiginosos sem Sintomas de Disfunção Vestibular à Vectoeletronistagmografia. 1996. 61p. Tese (Livre Docência) - Escola Paulista de Medicina, Universidade Federal de São Paulo, São Paulo, 1996.

3. CAOVILlA, H.H. - Auto-rotação cefálica no diagnóstico da disfunção vestibular. RBM-ORL Otologia, 57:8-11, 2000.
4. CAOVILLA, H.H.; GANANÇA, M.M. - Ganho, fase e assimetria à rotação cefálica ativa com Vorteq: limites normais. RBM-ORL, 3(4):209-12, 1996.

5. __ - Rotação Cefálica Ativa de altas freqüências: método simples e fisiológico para avaliação rápida e precisa da função vestibular. RBM-ORL, 4(1):158-63, 1997.

6. CAOVIlla, H.H. et al. - O Equilíbrio Corporal e seus Distúrbios. Parte II Lidando com o paciente vertiginoso. RBM-ORL, 4(2):47-51, 1997a.

7. _ - O Equilíbrio Corporal e seus Distúrbios. Parte V: O Valor da Nistagmografia Computadorizada. RBM-ORL, 4(5):158-63, 1997 b.

8. ENG, VORTEC and rotational chair user's manual version 4.5. Chatham, Micromedical technologies, 1995. 194p.

9. FINEBERG, R.; O'LEARY, D.P.; DAVIS, L.L. - Use of Active Head Movements for Computerized Vestibular Testing. Arch Otolaryngol Head Neck Surg 113:1063-5, 1987.

10. GANANÇA, M.M.; CAOVILLA, H.H. - Labirintopatias. ACTA-AWHO 10(1):4-16, 1991.

11. GANANÇA, M.M. (editor) - Vertigem tem Cura? O que aprendemos nos últimos 30 anos. São Paulo, Lemos Editorial, 1998. 301p.

12. GANANÇA, M.M. et al. - A Vertigem Explicada: I Diretrizes Diagnósticas. RBM Caderno de Vertigem 56, 1999. 20p.

13. - As etapas da equilibriometria, In: CAOVILLA, H.H. et al. Equilíbriometria Clinica. Série Otoneurológica. Vol. 1., São Paulo, Atheneu, 1999 pag. 41-114, 158p.

14. GANANÇA, M.M.; MUNHOZ, M.S.L.; CAOVILLA, H.H. - Utilidade clínica do exame otoneurológico. RBM-ORL Otologia 57:2-7, 2000.

15. GANANÇA, M.M. - Qual a prevalência da vertigem e outras tonturas de origem vestibular de acordo com a faixa etária? ACTA-AWHO 19(4):170-1, 2000.

16. GORDON, C.R. et al. - Nonspecific vertigo with normal otoneurological examination. The role vestibular laboratory tests. The Jlaryngol otology, 110:1133-7, 1996.

17. HAMID, M.A.; HUGHES, G.B.; KINNEY, S.E. - Criteria for diagnosing bilateral vestibular dysfundion. In: GRAHAM, M.D.; KEMMINK, J.L. ed. - The Vestibular System: Neurophysiologic and Clinical Research. New York, Raven, 1987. p. 115-8.

18. HIRVONEN, T.P. et al. - Changes in vestibulo-ocular.reflex of elderly people. Acta-otolaryngol (Stockh.), 529:108-10, 1997a(suppl).

19. HIRVONEN, T.P. et al. - Vestibulo-ocular reflex function as measured with the head autorotation test. Acta-otolaryngol (Stockh.), 1175):65762, 1997b.

20. KITSIGIANIS,G.; O'LEARY,D.P.; DAVIS,L. - Active head-movement analysis of cisplatin-induced vestibulotoxicity. Otolaryngol bead neck surg, 98:82-7, 1988.

21. MURPHY,T.P. - Vestibular auto-rotation and electronystagmography testing in patients with dizziness. Am jotology., 15(4):502-5, 1994.

22. MAUDONNET, O; FRANCIS, G.; MAUDONNET, E. - Prevalence of vestibular and cochlear diseases in 4825 pacients. Rev bras otorrinolaringol. 65(1):26-33, 1999.

23. MEULENBROEKS, A.A.W.M. et al. - Quantitative evaluation of the vestibular auto-rotation test (VAT) in normal subjects. Acta otolaryngol (Stockh), 520:327-33, 1995, suppl.

24. O'LEARY, D.P.; DAVIS,L.L.; KEVORKIAN, K.F. - Dynamic analysis of age-related responses of the vestibulo-ocular reflex. In: CAMPOS, M.C.A. (eds) - Inner Ear Pathobiology. Adv Otorhinolaryngol. Basel, Karger, 45:194-202, 1990.

25. O'LEARY, D.P.; DAVIS, L.L. - Vestibular auto-rotation testing of Meniere's disease. Otolaryngol head neck surg, 103(1):66-71, 1990.

26. O'LEARY, D.P.; DAVIS, L.L.; MACERI, D.R. - Vestibular auto-rotation test asymmetry analysis of acoustic neuromas. Otolaryngol head neck surg, 104(1):103-9, 1991.

27. SAADAT et al. - Comparison of vestibular auto-rotation and caloric testing. Otolaryngol head neck surg, 113(3):215-22, 1995.

28. TOMLINSON, R.D.;SAUNDERS, G.E.;SCHWARZ, D.W.F. - Analysis of human vestibulo-ocular reflex during active head moviments. Acta Otolaryngol., 90:184-190, 1980. 